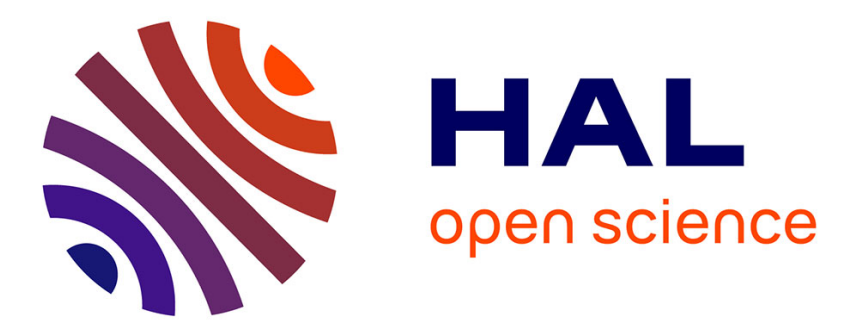

\title{
Analytical Calculation of the Instantaneous Power in a Current Carrying Superconducting Tube with Jc(B)
}

\author{
Bruno Douine, Kévin Berger, Jules E. Pienkos, Jean Lévêque, Denis Netter
}

\section{To cite this version:}

Bruno Douine, Kévin Berger, Jules E. Pienkos, Jean Lévêque, Denis Netter. Analytical Calculation of the Instantaneous Power in a Current Carrying Superconducting Tube with Jc(B). IEEE Transactions on Applied Superconductivity, 2008, 18 (3), pp. 1717-1723. 10.1109/TASC.2008.2000906 . hal00348410v2

\section{HAL Id: hal-00348410 \\ https://hal.science/hal-00348410v2}

Submitted on 8 Feb 2011

HAL is a multi-disciplinary open access archive for the deposit and dissemination of scientific research documents, whether they are published or not. The documents may come from teaching and research institutions in France or abroad, or from public or private research centers.
L'archive ouverte pluridisciplinaire HAL, est destinée au dépôt et à la diffusion de documents scientifiques de niveau recherche, publiés ou non, émanant des établissements d'enseignement et de recherche français ou étrangers, des laboratoires publics ou privés. 


\title{
Analytical Calculation of the Instantaneous Power in a Current Carrying Superconducting Tube With $J_{C}(B)$
}

\author{
Bruno Douine, Kévin Berger, Jules E. Pienkos, Jean Lévêque, and Denis Netter
}

\begin{abstract}
To use high-Tc superconductors for alternating current transport, it is important to evaluate $\mathrm{AC}$ losses. The influence of $J_{c}(B)$ on the self field losses in a superconductive tube fed by a transport current in incomplete penetration is studied for two reasons. First, superconducting power cables have a geometry that resembles a tube and second, for high-temperature superconductors, the variation of $J_{c}(B)$ is important especially for low magnetic fields like self field. An analytical calculation of the distribution of the magnetic field $B(r, t)$ by using a linearized law $J_{c}(B)$ is presented. From $B(r, t)$ one deduces $J(r, t)$ and $E(r, t)$. The analytical expressions of those were used to calculate the analytical instantaneous power $p(t)$. The losses in the superconducting tube are the average value of $p(t)$. They are numerically calculated and compared with the measurements taken on a sample whose characteristic $J_{c}(B)$ was previously measured. With the linear model, the calculated losses are closer to the measured losses than for the Bean model, but the $J_{c}(B)$ identification remains the main problem.
\end{abstract}

Index Terms-AC losses, critical current density, high-temperature superconductor (HTS).

\section{INTRODUCTION}

$\mathbf{H}$ IGH-CRITICAL-TEMPERATURE superconductors (HTS) used for transport of alternating electric current present lower electrical losses than resistive materials. AC losses have a deleterious effect on the cooling system and must be evaluated.

The analytical calculation of the AC losses in bulk superconductors was already presented in the following cases:

1) cylinder and plate in a variable external magnetic field with constant critical current density $J_{c}[1]$;

2) sheet in a variable external magnetic field with $J_{C}$ dependent on magnetic flux density $B[2]$;

3) cylinder and tube with transport current and constant $J_{c}$ [3], [4].

The theoretical model used for these calculations was proposed by Bean [5]. This model assumed that the critical current

Manuscript received August 24, 2007; revised January 16, 2008, January 28, 2008, February 25, 2008, and March 17, 2008. First published July 15, 2008; current version published September 4, 2008. This paper was recommended by Associate Editor J. Willis.

B. Douine, J. Lévêque, and D. Netter are with the Groupe de Recherche en Electrotechnique et Electronique de Nancy, University of Nancy, 54506 Vandoeuvre, France (e-mail: bruno.douine@green.uhp-nancy.fr; jean.leveque@green.uhp-nancy.fr; denis.netter@green.uhp-nancy.fr).

$\mathrm{K}$. Berger is with the Grenoble Electrical Engineering Laboratory, 38042 Grenoble, France (e-mail: Kevin.Berger@g2elab.inpg.fr).

J. E. Pienkos is with the Center of Advanced Power Systems, Tallahassee, FL 32310 USA (e-mail: pienkos@caps.fsu.edu).

Digital Object Identifier 10.1109/TASC.2008.2000906 density at any point of the sample can only take one of three constant values, the critical current density $J_{c},-J_{c}$, and zero. That is the critical state model (CSM).

Superconducting power cables have a geometry that resembles a tube comprised of superconducting filaments in a copper or silver matrix. Despite the inhomogeneous nature of these filament-matrix cables, the losses are calculated with the monoblock model using average current densities and thickness [6]. $J_{c}(B)$ varies for low magnetic field in HTS [7], like in the case of transport current. Large variation of $J_{c}(B)$ leads to greater variation of $\mathrm{AC}$ losses and penetration current $I_{p}$.

Previous work has numerically calculated the AC losses as a function of $J_{c}(B)$ [8]-[10]. AC loss analytical calculations using variable $J_{c}(B)$ give exact results without numerical error. Three ways allows one to analytically calculate $\mathrm{AC}$ losses, using pointing vector [2], the magnetic flux [3], [5], [11]-[14], or the instantaneous power $p(t)$ [1], [4], [15]-[17]. Knowing $p(t)$ also allows one to calculate thermal stability and thermal response of superconducting systems [18]-[20]. In this paper, analytical calculation of the instantaneous power in a current carrying a superconducting tube and the calculation of $J_{c}(B)$ influences on the $\mathrm{AC}$ losses are studied. These calculations are made thanks to the linearized $J_{c}(B)$ law; therefore, the AC losses are called the linear model losses $P_{L}$. In [7], the influence of $J_{c}(B)$ on $I_{p}$ is presented. The experimentalists showed how to obtain a linear law for $J_{c}(B)$ starting from the measured $J_{c M}$ according to an external magnetic field $B_{\text {ext }}$.

Expressions of the steady state distributions of $B(r, t)$, $J(r, t)$, electric field $E(r, t)$, and instantaneous power $p(t)$ for incomplete penetration (current of transport $i<I_{p}$ ) are presented. The losses $P_{L}$ are calculated by numerically integrating $p(t)$ over one period. Finally a comparison of the calculated values versus the loss measurements is examined.

\section{Magnetic Field, CuRRent Density, AND Electric FIELD DISTRIBUTIONS}

First, in Section II-A, the framework of this study is presented. Next, in Section II-B, previous results [7] necessary to comprehension of continuous rating are discussed. Then, in Sections II-C and II-D, the new calculations of AC losses are presented. In these last two sections, calculations were performed from $I_{\max }$ to negative $I_{\max }$ on half the periodic curve, thus the current is always decreasing. Following this decrease, there are two cases, the first when $i(t)$ is positive and the second during which $i(t)$ is negative. In these two cases $B(r, t), J(r, t)$, and $E(r, t)$ are different and are presented separately. 


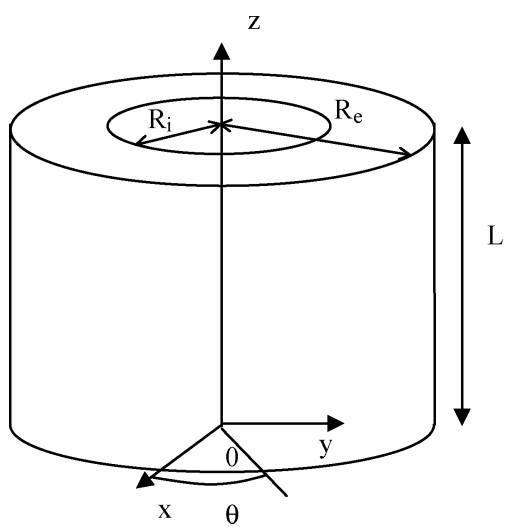

Fig. 1. Diagram of the studied superconducting tube.

\section{A. Frame of the Theoretical Study}

The case of a superconductive tube with transport current $i(t)$ is studied. The dimensions of the tube are internal radius $R_{\text {in }}$, external radius $R_{e}$, section $S=\pi\left(R_{e}^{2}-R_{\text {in }}^{2}\right)$, and length $h$, seen in Fig. 1. The z-axis represents the axis of the tube.

The electromagnetic behaviour of a superconductor is governed by the Maxwell equations

$$
\begin{aligned}
& \overrightarrow{\operatorname{rot}} \vec{E}=-\frac{\partial \vec{B}}{\partial t} \\
& \operatorname{rot} \vec{B}=\mu_{0} \cdot \vec{J}
\end{aligned}
$$

For the superconducting material, we assume the approximation

$$
B=\mu_{0} \cdot H
$$

$E(J)$ is given by the CSM and defines the relation between the electric field $E$ and the current density $J$

$$
\begin{aligned}
& E \neq 0: J= \pm J_{c} \\
& E=0:-J_{c}<J<+J_{c} .
\end{aligned}
$$

Due to symmetry and the current density being oriented along the z-axis, $B(r, t), J(r, t)$, and $E(r, t)$ have only one component

$$
\begin{aligned}
\vec{B}(r, t) & =B(r, t) \cdot \vec{u}_{\theta}, \vec{J}(r, t) \\
& =J(r, t) \cdot \vec{u}_{z}, \vec{E}(r, t)=E(r, t) \cdot \vec{u}_{z} .
\end{aligned}
$$

The tube is supplied by a periodic current $i(t)(\operatorname{period} T)$ (Fig. 2) between $I_{\max }$ and negative $I_{\max }$. The time wave form of the current $i(t)$ is unimportant for the calculations of the magnetic field distribution or the losses for the CSM [21].

\section{B. Transient State $(0<t<T / 4)$ and the Full Penetration Current $I_{p}$}

The transient state corresponds to the first rise of the current $i(t)(0<t<T / 4)$. This study has already been published [7]

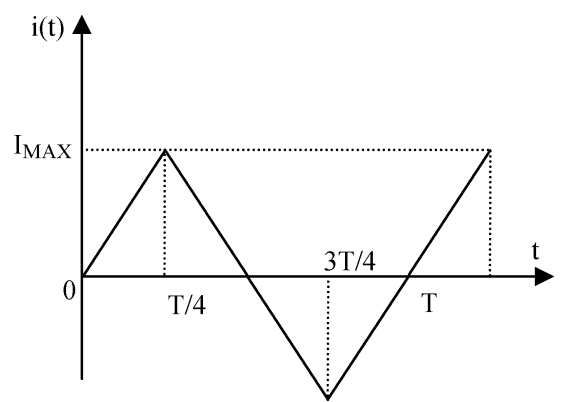

Fig. 2. Periodic current supply to the studied sample.

but it is necessary to review some calculations to understand this paper fully.

By considering (2) and (4), one obtains the following differential equation:

$$
\frac{1}{r} \frac{\partial(r \cdot B(r, t)}{\partial r}=\mu_{0} \cdot J_{c}(B)
$$

On the external radius, there is the following boundary condition:

$$
B\left(R_{e}, t\right)=b_{e}(t)=\frac{\mu_{0} \cdot i(t)}{2 \pi R_{e}} .
$$

Equation (5) has a relatively simple analytical solution with a linear $J_{c}(B)$. With another $J_{c}(B)$, the solutions of the $J(r, t)$, $B(r, t)$, and $E(r, t)$ distribution calculations are too complex to be useful. So, to allow an analytical calculation, $J_{c}(B)$ is linearized

$$
J_{c}(B)=\frac{J_{c 0}\left(B_{j 0}-|B|\right)}{B_{j 0}} .
$$

So (5) becomes

$$
\frac{1}{r} \frac{\partial(r \cdot B(r, t))}{\partial r}=\mu_{0} J_{c 0}\left(1-\frac{|B(r, t)|}{B_{j 0}}\right) .
$$

When the current rises, the current density penetrates from the external radius $R_{e}$, toward the internal radius $R_{\text {in }}$, and its direction is the $\mathrm{z}$-axis.

During the transient state, $i(t)>0, B(r, t)>0$, and (7) becomes

$$
\frac{1}{r} \frac{\partial(r \cdot B(r, t))}{\partial r}=\mu_{0} \cdot J_{c}(B)=\mu_{0} J_{c 0}\left(1-\frac{B(r, t)}{B_{j 0}}\right) .
$$

Considering (8) and (6), one deduces $B(r, t)$

$$
\begin{aligned}
& B(r, t)=\frac{B_{j 0}\left(\mu_{0} \cdot J_{c 0} \cdot r-B_{j 0}\right)}{\mu_{0} \cdot J_{c 0} \cdot r} \\
& \quad+\frac{\exp \left(\frac{\mu_{0} \cdot J_{c 0} \cdot\left(R_{e}-r\right)}{B_{j 0}}\right)\left(B_{j 0}^{2}+\mu_{0} \cdot J_{c 0} \cdot R_{e}\left(b_{e}(t)-B_{j 0}\right)\right)}{\mu_{0} \cdot J_{c 0} \cdot r} .
\end{aligned}
$$


At $t=T / 4\left(i(T / 4)=I_{\max }, b_{e}(T / 4)=\mu_{0} \cdot I_{\max } / 2 \pi R_{e}=\right.$ $\left.B_{e \max }\right)$, the magnetic flux density penetrates in the tube up to $r=r_{s}$ and is called $B_{\max }(r)$

$$
\begin{aligned}
r_{S}<r<R_{e}: B_{\max }(r)=\frac{B_{j 0}\left(\mu_{0} \cdot J_{c 0} \cdot r-B_{j 0}\right)}{\mu_{0} \cdot J_{c 0} \cdot r} \\
+\frac{\left(B_{j 0}^{2}+\mu_{0} \cdot J_{c 0} \cdot R_{e}\left(B_{e \max }-B_{j 0}\right)\right) \exp \left(\frac{\mu_{0} \cdot J_{c 0} \cdot\left(R_{e}-r\right)}{B_{j 0}}\right)}{\mu_{0} \cdot J_{c 0} \cdot r} .
\end{aligned}
$$

$r_{S}$ is obtained with $B_{\max }\left(r_{S}\right)=0$

$$
r_{S}=\frac{B_{j 0}}{\mu_{0} \cdot J_{c 0}}\left(1+W\left(X_{1}(T / 4)\right)\right)
$$

with $X_{1}(t)=-\left(\left(B_{j 0}^{2}+\mu_{0} J_{c 0} R_{e}\left(b_{e}(t)-\right.\right.\right.$ $\left.\left.\left.B_{j 0}\right)\right) / B_{j 0}^{2}\right) \exp \left(\left(\mu_{0} J_{c 0} R_{e} / B_{j 0}\right)-1\right)$, where $X_{1}>0$ and $W\left(X_{1}\right)$ is Lambert's $W$-function. This function, also called the omega function, is the inverse function of $f(W)=W \cdot \exp (W)[22]$.

With the CSM, $i(t)$ must remain lower than $I_{p}$ so that the material remains superconductive. $I_{p}$ depends on the law $J_{c}(B)$ of the material [7].

If the current penetration is complete $r_{s}=R_{\text {in }}$ and $i(t)=$ $I_{P}$. Using $B(r, t)$ one finds $I_{p}$ because $B\left(r=R_{\mathrm{in}}, b_{e}(t)=\right.$ $\left.\mu_{0} I_{P} / 2 \pi R_{e}\right)=0$

$$
\begin{aligned}
I_{p}= & \frac{2 \pi \cdot B_{j 0}}{\mu_{0}^{2} \cdot J_{c 0}} \cdot\left(\mu_{0} \cdot J_{c 0} \cdot R_{e}-B_{j 0}\right)+\frac{2 \pi \cdot B_{j 0}}{\mu_{0}^{2} \cdot J_{c 0}} \\
& \cdot\left(B_{j 0}-\mu_{0} \cdot J_{c 0} \cdot R_{\text {in }}\right) \cdot \exp \left(\frac{\mu_{0} \cdot J_{c 0} \cdot\left(R_{e}-R_{\text {in }}\right)}{B_{j 0}}\right) .
\end{aligned}
$$

\section{First Quarter of Period of the Continuous Rating $(T / 4<t<T / 2)$ and $i(t)>0$}

After the first current penetration, the current decreases and the current density has a value of negative critical current density $\left(\vec{J}=-J_{C}(B) \cdot \vec{u}_{z}\right)$ with respect to the circumference (Fig. 3), for $c_{1}(t)<r<R_{e}$, opposed to that of the center where $\vec{J}=$ $J_{C}(B) \cdot \vec{u}_{z}$ and $B(r, t)=B_{\max }(r)$ for $r_{s}<r<c_{1}(t)$. The trapped magnetic field $B_{\max }(r)$ is constant for $t>T / 4$ and $r_{s}<r<c_{1}(t)$.

So, (8) becomes

$$
\frac{1}{r} \frac{\partial(r \cdot B(r, t))}{\partial r}=-\mu_{0} \cdot J_{c}(B)=-\mu_{0} J_{c 0}\left(1-\frac{B(r, t)}{B_{j 0}}\right) .
$$

As long as $i(t)>0$ and $B(r, t)>0$, one calculates $B(r, t)$ by solving (11) with (6)

$$
\begin{aligned}
& c_{1}(t)<r<R_{e}: B(r, t)=\frac{B_{j 0}\left(\mu_{0} \cdot J_{c 0} \cdot r+B_{j 0}\right)}{\mu_{0} \cdot J_{c 0} \cdot r} \\
& -\frac{e^{\frac{\mu_{0} \cdot J_{c 0} \cdot\left(r-R_{e}\right)}{B_{J 0}}}\left(B_{j 0}^{2}-\mu_{0} \cdot J_{c 0} \cdot R_{e}\left(b_{e}(t)-B_{j 0}\right)\right)}{\mu_{0} \cdot J_{c 0} \cdot r} .
\end{aligned}
$$

To calculate $c_{1}(t)$, we know that $B(r, t)$ is spatially continuous at $r=c_{1}(t)$, as shown in (13), at the top of the next page.

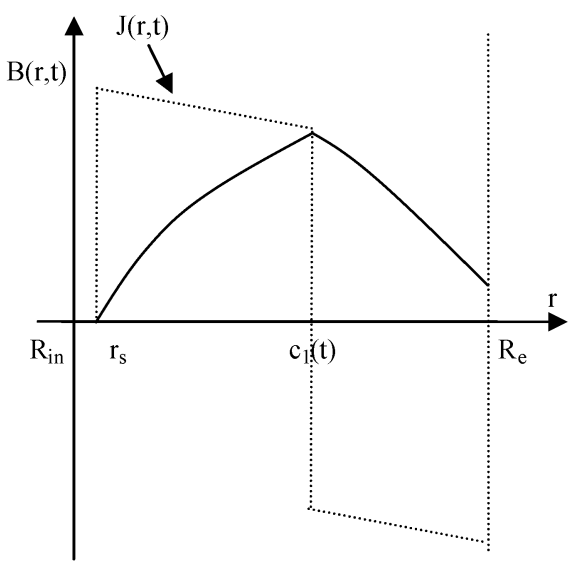

(a)

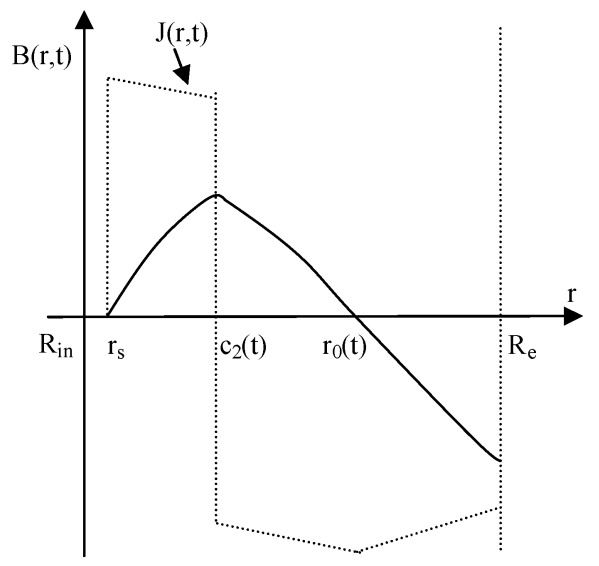

(b)

Fig. 3. Distributions of $B(r, t)$ and $J(r, t)$ versus $r$ with the linear model. (a) $i(t)>0$; (b) $i(t)<0$.

The electric field distribution is important to calculate the AC losses because

$$
P_{L}=\frac{1}{T} \int_{T} \int_{V} \vec{E} \cdot \vec{J} \cdot d v \cdot d t \quad(V \text { is the tube volume }) .
$$

One obtains $E(r, t)$ with (1) that becomes

$$
\frac{\partial E}{\partial r}=\frac{\partial B}{\partial t} .
$$

We can calculate $E(r, t)$ using the boundary condition $E[r=$ $\left.c_{1}(t), t\right]=0$

$$
\text { For } \begin{aligned}
c_{1}(t)< & r<R_{e}: \\
E(r, t)= & \frac{\mu_{0}}{2 \pi} \cdot \frac{d i(t)}{d t} \cdot e^{-\frac{\mu_{0} \cdot J_{c 0} \cdot R_{e}}{B_{J 0}}} \\
& \times\left[E_{i}\left(\frac{\mu_{0} \cdot J_{c 0} \cdot r}{B_{j 0}}\right)-E_{i}\left(\frac{\mu_{0} \cdot J_{c 0} \cdot c_{1}(t)}{B_{j 0}}\right)\right] .
\end{aligned}
$$

$E_{i}(z)=-\int_{-z}^{+\infty}\left(e^{-t} / t\right) d t$ is an exponential integral function. 


$$
\begin{aligned}
B_{\max }\left(c_{1}(t)\right)= & B\left(c_{1}(t), t\right) \\
= & \frac{B_{j 0}\left(\mu_{0} \cdot J_{c 0} \cdot c_{1}(t)+B_{c 0}\right)}{\mu_{0} \cdot J_{c 0} \cdot c_{1}(t)} \\
& -\frac{\mu^{\frac{\mu_{0} \cdot J_{c 0} \cdot\left(c_{1}(t)-R_{e}\right)}{B_{c 0}}}\left(B_{j 0}^{2}-\mu_{0} \cdot J_{c 0} \cdot R_{e}\left(b_{e}(t)-B_{j 0}\right)\right)}{\mu_{0} \cdot J_{c 0} \cdot c_{1}(t)} \\
c_{1}(t)= & R_{e}+\frac{B_{j 0}}{\mu_{0} J_{c 0}} \\
& \times \log \left[\frac{B_{j 0}^{2}+\sqrt{B_{j 0}^{2}\left(b_{e}(t)-B_{e \max }\right)-\mu_{0} J_{c 0} R_{e}\left(B_{e \max } b_{e}(t)-B_{e \max } B_{j 0}-B_{j 0} b_{e}(t)+B_{j 0}^{2}\right)}}{B_{j 0}^{2}+\mu_{0} J_{c 0} R_{e}\left(B_{j 0}-b_{e}(t)\right)}\right]
\end{aligned}
$$

\section{Second Quarter of Period of the Continuous Rating $(T / 2<t<3 T / 4)$ and $i(t)<0$}

For $T / 2<t<3 T / 4, i(t)<0, B(r, t)$ is positive in the internal portion of the tube $\left(c_{2}(t)<r<r_{0}(t)\right)$ and negative in the external portion $\left(r_{0}(t)<r<R_{e}\right)$ (Fig. 3). For $r=r_{0}$ and for all $t, B\left(r=r_{0}, t\right)=0$. If $B(r, t)<0$, the equation can be solved as stated previously (11). If $B(r, t)<0$, the equation used is

$$
\frac{1}{r} \frac{\partial(r \cdot B(r, t))}{\partial r}=-\mu_{0} \cdot J_{c}(B)=-\mu_{0} J_{c 0}\left(1+\frac{B(r, t)}{B_{j 0}}\right) .
$$

First, the $B(r, t)$ profile with respect to the radius is presented.

For $r_{S}<r<c_{2}(t), B(r, t)=B_{\max }(r)$ is the trapped magnetic flux density.

For $c_{2}(t)<r<r_{0}(t)$, considering (11) and that $B\left(r_{0}(t)\right)=$ 0 , one deduces $B(r, t)$

$$
\begin{aligned}
0 & <B(r, t) \\
& =\frac{B_{j 0}}{\mu_{0} J_{c 0} r} \cdot\left[B_{j 0}+\mu_{0} J_{c 0} r-e^{g(r)-f_{2}(t)}\left(B_{j 0}+\mu_{0} J_{c 0} r_{0}(t)\right)\right]
\end{aligned}
$$

with $f_{2}(t)=\mu_{0} J_{c 0} r_{0}(t) / B_{j 0}$ and $g(r)=\mu_{0} J_{c 0} r / B_{j 0}$.

For $r_{0}(t)<r<R_{e}$, considering (15) and (6), one deduces $B(r, t)$

$$
\begin{aligned}
& 0>B(r, t) \\
& \begin{aligned}
=\frac{1}{\mu_{0} J_{c 0} r} \cdot & {\left[B_{j 0}\left(B_{j 0}-\mu_{0} J_{c 0} r\right)+e^{A-g(r)}\right.} \\
& \left.\times\left(-B_{j 0}^{2}+\mu_{0} J_{c 0} R_{e}\left(B_{j 0}+b_{e}(t)\right)\right)\right] .
\end{aligned}
\end{aligned}
$$

Second, the important positions $r_{0}(t)$ and $c_{2}(t)$ are calculated. After solving (17), one finds $r_{0}(t)$ because $B\left(r_{0}(t)\right)=0$

$$
r_{0}(t)=\frac{B_{j 0}}{\mu_{0} J_{c 0}} \log \left[1+W\left(X_{2}\right)\right]
$$

with $X_{2}(t)=\left(\left(-B_{j 0}^{2}+\mu_{0} J_{c 0} R_{e}\left(b_{e}(t)+B_{j 0}\right)\right) / B_{j 0}^{2}\right) \exp (A-$ 1), $A=\mu_{0} J_{c 0} R_{e} / B_{j 0}$.

Since $B(r, t)$ is continuous, this allows $c_{2}(t)$ to be calculated

$$
c_{2}(t)=\frac{B_{j 0}}{\mu_{0} \cdot J_{c 0}} \log \left[\frac{2 B_{j 0}^{2} \cdot \exp \left(1+W\left(X_{2}\right)\right)+\sqrt{F(t)}}{B_{j 0}^{2} \cdot\left(2+W\left(X_{2}\right)\right)}\right]
$$

with

$$
\begin{aligned}
F(t)= & B_{j 0}^{4} \cdot \exp \left(2 \cdot\left(1+W\left(X_{2}\right)\right)\right) \\
& -2 \cdot B_{j 0}^{2} \cdot \exp \left(1+A+W\left(X_{2}\right)\right) \\
& \cdot\left[\left(B_{j 0} \cdot\left(B_{j 0}-\mu_{0} \cdot J_{c 0} \cdot R_{e}\right)\right)+\mu_{0} \cdot J_{c 0} \cdot R_{e} \cdot B_{e \max }\right] \\
& +e^{2 A} \cdot\left[\left(B_{j 0} \cdot\left(B_{j 0}-\mu_{0} \cdot J_{c 0} \cdot R_{e}\right)\right)-\mu_{0} \cdot J_{c 0} \cdot R_{e} \cdot b_{e}(t)\right] \\
& \cdot\left[\left(B_{j 0} \cdot\left(B_{j 0}-\mu_{0} \cdot J_{c 0} \cdot R_{e}\right)\right)+\mu_{0} \cdot J_{c 0} \cdot R_{e} \cdot B_{e \max }\right]
\end{aligned}
$$

and $f_{1}(t)=\mu_{0} J_{c 0} c_{2}(t) / B_{j 0}$.

Third, similar to Section II-C, one obtains $E(r, t)$ from $B(r, t)$

For $c_{2}(t)<r<r_{0}(t)$ :

$E(r, t)=\mu_{0} J_{c 0} r_{0}(t) \frac{d r_{0}(t)}{d t} \cdot e^{-f_{2}(t)}\left[E_{i}(g(r))-E_{i}\left(f_{1}(t)\right)\right]$

For $r_{0}(t)<r<: R_{e}$,

$$
\begin{aligned}
E(r, t)=\mu_{0} \cdot J_{c 0} \cdot r_{0}(t) \frac{d r_{0}(t)}{d t} & \\
\cdot e^{-f_{2}(t)} & {\left[\left(E_{i}\left(f_{2}(t)\right)-E_{i}\left(f_{1}(t)\right)\right)\right.} \\
& \left.+\frac{e^{-\left(A+f_{2}(t)\right)}}{2 \pi J_{c 0}}\left(E_{i}(-g(r))-E_{i}\left(-f_{2}(t)\right)\right)\right] .
\end{aligned}
$$

\section{Analytical InStantaneous Power AND Numerical AC LOSS CALCULATIONS}

The constant $J_{C}$ analytical calculations of AC losses $P$, already presented by several authors [1], [3], [6], [11]-[14], are based on a common demonstration. 
First, $P$ is defined as

$$
\begin{aligned}
P & =\frac{1}{T} \int_{T} p(t) \cdot d t=\frac{1}{T} \int_{T} \int_{V} \vec{E} \cdot \vec{J} \cdot d v \cdot d t \\
& =\frac{4 \pi h}{T} \int_{T / 4}^{3 T / 4} \int_{R_{\text {in }}}^{R_{e}} E(r, t) J(r, t) \cdot r \cdot d r \cdot d t .
\end{aligned}
$$

Second, $E(r, t)$ is not null and $\partial E / \partial r=\partial B / \partial t$ only for $c(t)<r<R_{e}$, so

$$
\begin{aligned}
P & =\frac{4 \pi h}{T} \int_{T / 4}^{3 T / 4} \int_{r_{S}}^{R_{e}} \int_{c(t)}^{r} \frac{\partial B\left(r^{\prime}, t\right)}{\partial t} d r^{\prime} \cdot J(r, t) \cdot r \cdot d r \cdot d t \\
& =\frac{4 \pi h}{T} \int_{r_{s}}^{R_{e}} \int_{T / 4}^{3 T / 4} \int_{c(t)}^{r} \frac{\partial B\left(r^{\prime}, t\right)}{\partial t} d r^{\prime} \cdot J(r, t) \cdot d t \cdot r \cdot d r .
\end{aligned}
$$

Third, if $J_{C}$ is assumed constant [1], [3], [6], [11]-[14], $P$ becomes

$$
\begin{aligned}
P & =\frac{4 \pi h J_{C}}{T} \int_{r_{s}}^{R_{e}} \int_{T / 4}^{3 T / 4} \int_{c(t)}^{r} \frac{\partial B\left(r^{\prime}, t\right)}{\partial t} d r^{\prime} \cdot d t \cdot r \cdot d r \\
& =\frac{4 \pi h J_{C}}{T} \int_{r_{s}}^{R_{e}} \int_{c(t)}^{r} \int_{T / 4}^{T / 4} \frac{\partial B\left(r^{\prime}, t\right)}{\partial t} d t \cdot d r^{\prime} \cdot r \cdot d r .
\end{aligned}
$$

Fourth, $B(r, t=T / 4)=-B(r, t=3 T / 4)=B_{\max }(r)$ and $c(t=T / 4)=r_{S}$, so

$$
P=\frac{8 \pi h J_{C}}{T} \int_{r_{s}}^{R_{e}} \int_{r_{S}}^{r} B_{\max }\left(r^{\prime}\right) d r^{\prime} \cdot r \cdot d r
$$

This last formula is simple and useful with constant $J_{C}$ but not useful with $J_{C}(B)$. In this case to calculate AC losses, $p(t)$ has to be calculated using

$$
p(t)=\int_{V} \vec{E} \cdot \vec{J} \cdot d v=2 \pi h \int_{R_{\text {in }}}^{R_{e}} E(r, t) \cdot J(r, t) \cdot r \cdot d r .
$$

As in Section II, $p(t)$ is expressed differently dependent upon whether $i(t)$ is positive or negative.

Thanks to the expressions of $B(r, t), E(r, t)$, and $J(r, t)$ :

1) $i(t)>0$

$$
\begin{aligned}
p(t)=2 \pi h \int_{c_{1}(t)}^{R_{e}} E(r, t) \cdot J(r, t) \cdot r \cdot d r \\
p(t)=\frac{h \cdot e^{-2 A}}{2 \cdot \pi \cdot \mu_{0} \cdot J_{c 0}} \frac{d i}{d t} \\
\times\left[2 \cdot \pi \cdot B_{j 0}^{2}\left(\exp \left(A+f_{1}(t)\right)-\exp (2 A)\right)\right. \\
\quad+e^{A}\left[2 \cdot \pi \cdot B_{j 0}^{2}-\mu_{0}^{2} \cdot J_{c 0} \cdot i(t)\right] \cdot\left[E_{i}\left(f_{1}(t)\right)-E_{i}(A)\right] \\
\\
+\left[\mu_{0}^{2} \cdot J_{c 0} \cdot i(t)-2 \cdot \pi \cdot B_{j 0}\left(B_{j 0}+\mu_{0} \cdot J_{c 0} \cdot R_{e}\right)\right] \\
\\
\left.\quad \cdot\left[E_{i}\left(2 f_{1}(t)\right)-E_{i}(2 A)\right]\right]
\end{aligned}
$$

2) $i(t)<0$, as shown in the equation at bottom of page.

The two expressions of $p(t)$ are complex and do not allow one to calculate the losses $P_{L}$ that is the $p(t)$ average value, which require an integration with respect to time $t$. Thus, the calculation of $P$ is done by numerical integration

$$
P_{L}=\frac{1}{T} \int_{T} p(t) \cdot d t \approx \frac{1}{N} \cdot \sum_{i=1}^{N} p(i . \Delta t)
$$

where

$\Delta t \quad$ is the time slot;

$N=100 \quad$ is the quantity of time slots;

$T=N . \Delta t$.

$$
\begin{aligned}
& p(t)=2 \pi h \int_{c_{2}(t)}^{r_{0}(t)} E(r, t) \cdot J(r, t) \cdot r \cdot d r+2 \pi h \int_{r_{0}(t)}^{R_{e}} E(r, t) \cdot J(r, t) \cdot r \cdot d r \\
& p(t)=\frac{h \cdot e^{-2 f_{2}}}{4 \cdot \pi \cdot \mu_{0}}\left[8 \pi ^ { 2 } B _ { j 0 } r _ { 0 } \frac { d r _ { 0 } } { d t } \left[B_{j 0}\left[e^{f_{1}+f_{2}}-e^{2 f_{2}}+E_{i}\left(2 f_{2}\right)-E_{i}\left(2 f_{1}\right)+e^{2 f_{2}} \cdot\left(E_{i}\left(f_{1}\right)-E_{i}\left(f_{2}\right)\right)\right]\right.\right. \\
& \left.+\mu_{0} J_{c 0} r_{0}(t)\left(E_{i}\left(f_{1}\right)-E_{i}\left(f_{2}\right)\right)\right] \\
& +\frac{1}{J_{c 0}}\left[2 \frac { d i } { d t } \left[2 \cdot \pi \cdot B_{j 0}^{2}\left(e^{A+f_{2}}-e^{2 f_{2}}\right)-e^{A+2 f_{2}}\left(E_{i}(-A)-E_{i}\left(-f_{2}\right)\right) \cdot\left(2 \cdot \pi \cdot B_{j 0}^{2}-\mu_{0}^{2} J_{c 0} i(t)\right)\right.\right. \\
& \left.+e^{2\left(A+f_{2}\right)}\left(E_{i}(-2 A)-E_{i}\left(-2 f_{2}\right)\right)\left(2 \pi B_{j 0}\left(B_{j 0}-\mu_{0} J_{c 0} R_{e}\right)-\mu_{0}^{2} J_{c 0} i(t)\right)\right] \\
& +4 \pi J_{c 0} r_{0} \frac{d r_{0}}{d t}\left[E_{i}\left(f_{1}\right)-E_{i}\left(f_{2}\right)\right] \cdot\left[e^{f_{2}}\left(2 \pi B_{j 0}\left(B_{j 0}-\mu_{0} J_{c 0} R_{e}\right)-\mu_{0}^{2} J_{c 0} i(t)\right)\right. \\
& \left.\left.+e^{A}\left(\mu_{0}^{2} J_{c 0} i(t)-2 \pi B_{j 0}\left(B_{j 0}-\mu_{0} J_{c 0} R_{e}\right)\right)\right]\right]
\end{aligned}
$$




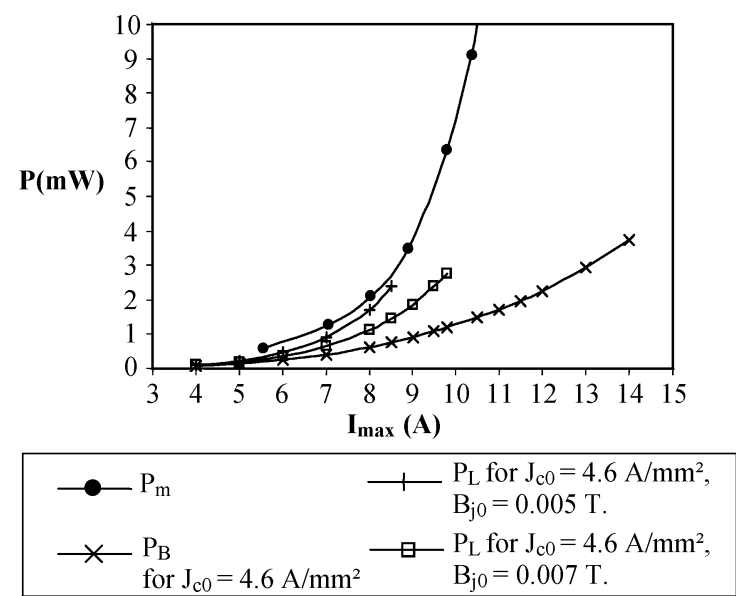

(a)

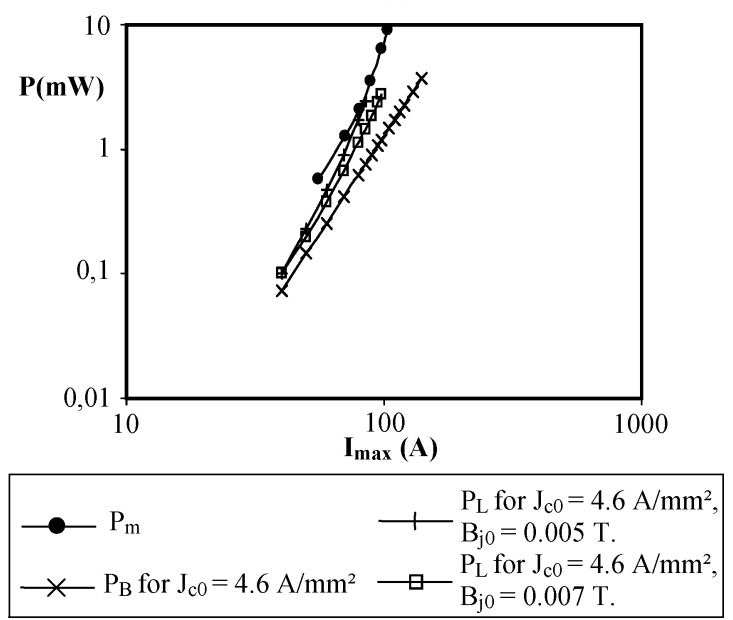

(b)

Fig. 4. Compared AC losses between the Bean model $P_{B}$, linear model $P_{L}$, and measured data $P_{m}$ with (a) linear scale and (b) $\log -\log$ scale.

\section{Comparison BetweEn MEASUREMENT AND CALCUlation}

The experimental tests were made using a cylindrical current lead of BiSCCO. The dimensions of this sample are: $R_{\text {in }}=$ $3.8 \mathrm{~mm}, R_{e}=5 \mathrm{~mm}, S=33 \mathrm{~mm}^{2}$, and $h=11.7 \mathrm{~cm}$, as seen in Fig. 1. In [7], the average characteristic $J_{\mathrm{mc}}\left(B_{\text {ext }}\right)$ curve for the superconductive tube was presented. AC loss calculations require the use of local data. By linearizing the $J_{\mathrm{mc}}\left(B_{\text {ext }}\right)$ curve, one may obtain a local data with the approximation

$$
J_{c}(B)=\frac{4.6 .10^{6}(0.007-|B|)}{0.007} \mathrm{~A} / \mathrm{m}^{2} .
$$

With (10) and the local approximation, one obtains $I_{p}=98 \mathrm{~A}$. AC losses were then measured on the experimental sample. The experimental losses were compared with theoretical calculations. The measured losses $P_{m}$, the losses calculated using the Bean model $P_{B}$, and the losses calculated with our linear model $P_{L}$ versus $I_{\max }$ are presented in Fig. 4. AC losses can be calculated by the Bean model or the linear model when $I(t)$ is less than $I_{p}$. For this case, $I_{p}$ is equal to $I_{c}$ for the Bean model and $I_{p}$ is less than $I_{c}$ for the linear model [7]. The Bean model uses one critical current density and is chosen as the value of $J_{c}(B)$ when $B=0$. With this value of $J_{c}$, the Bean model critical current is equal to $I_{c}=140 \mathrm{~A}$. One observes in Fig. 4 that the Bean model calculations for $\mathrm{AC}$ losses are lower than the measured losses. The difference between the Bean model calculations and the measured values becomes greater when $I_{\max }$ increases. The linear model is an improvement on the Bean model and compares more accurately with the measured losses. However, the linear model with the value calculated from (19) can still be improved by slightly changing the slope of $J_{c}(B)$. Thus we chose to vary one of the parameters of $J_{c}(B)$ so that the curve of the calculated losses approaches the measured losses. The difference in the new $J_{c}(B)$ may be the result of the averaged slope being used for local data [7]. Changing $B_{j 0}$ from $0.007 \mathrm{~T}$ to $0.005 \mathrm{~T}$, when $J_{c}(B)$ is zero, drops $I_{p}$ to $85 \mathrm{~A}$ from $98 \mathrm{~A}$ and one obtains a better agreement between the calculation and measurements of the losses. When $B_{j 0}$ decreases, the losses decrease for the maximum value of $I_{\max }$, or $I_{p}$. However, the decrease in $B_{j 0}$ has a greater effect on $I_{\max }$ less than $I_{p}$, and the overall losses increase. It is important to note that the $I_{p}$ limits the validity of the AC loss calculations by creating a maximum boundary for $I_{\max }$ [7]. Experimentally, the current fully penetrates the sample when $I_{\max } \geq 85 \mathrm{~A}$ and below is not in a complete current penetration state. This full penetration state assumption is derived from the theoretical curve of $\mathrm{AC}$ losses when $B_{j 0}$ equals $5 \mathrm{mT}$. In the last case, the Bean model and the linear model are unable to calculate AC losses for $I_{\max }$ greater than $I_{p}$. Choosing parameters of $J_{c}(B)$ are important because local $J_{c}(B)$ values must be calculated for AC losses. Therefore, the choice of parameters is important for the calculation of the losses, in particular in self magnetic fields, and it is even more difficult to identify $J_{c}(B)$ [7]. The difficulty of this choice explains why many researchers still work on this subject.

\section{CONCLUSION}

The influence of $J_{c}(B)$ on the losses in a superconductive tube fed by a transport current in incomplete current penetration has been studied. We developed an analytical calculation of the distribution of the magnetic field $B(r, t)$ by using a linearized law $J_{c}(B)$. From $B(r, t)$, one deduces $J(r, t)$ and $E(r, t)$. These analytical expressions were used to calculate the instantaneous power $p(t)$ of the tube. The calculation of the losses $P$ is done by numerically averaging $p(t)$ because the expression of $p(t)$ is too complex. These calculated losses are compared with the experimental measurements taken on a sample using characteristic $J_{m c}(B)$ that was previously measured [7]. Closer examination of material properties allows a better value of $J_{c}(B)$ which describes the measured losses more accurately. Future work includes the identification of the local law $J_{c}(B)$ using experimental data and the linearization of this law to calculate AC losses [7].

\section{REFERENCES}

[1] W. J. Carr, AC Loss and Macroscopic Theory of Superconductors. New York: Gordon and Breach, 1983, p. 158.

[2] G. Fournet and A. Mailfert, "Pénétration de l'induction, champ électrique et pertes dans les supraconducteurs de seconde espèce impurs présentant un courant de surface," J. Phys., vol. 31, pp. 357-367, Apr. 1970.

[3] M. N. Wilson, Superconducting Magnets. Oxford, U.K.: Oxford Science, 1983 , p. 335 
[4] B. Douine, D. Netter, J. Leveque, and A. Rezzoug, "AC losses in a BSCCO current lead: Comparison between calculation and measurement," IEEE Trans. Appl. Supercond., vol. 12, no. 1, pp. 1603-1606, Mar. 2002.

[5] C. P. Bean, "Magnetization of high-field superconductors," Rev. Mod. Phys., pp. 31-39, Jan. 1964

[6] M. Daumling, "AC loss in two ac carrying superconducting concentric tubes-The duo block model," Physica C, vol. 403, pp. 57-59, 2004.

[7] B. Douine, K. Berger, J. Leveque, D. Netter, and A. Rezzoug, "Influence of $\mathrm{Jc}(\mathrm{B})$ on the full penetration current of superconducting tube," Physica C, vol. 443, pp. 23-28, 2006.

[8] J. Paasi, J. Lehtonen, M. Lahtinen, and L. Kettunen, "Computation of AC losses in HTS," Physica C, vol. 310, pp. 62-66, 1998.

[9] D.-X. Chen, A. Sanchez, and E. Pardo, "Transport ac loss of a superconducting cylinder with field and radius dependent critical-current density," Superconductor Sci. Technol., vol. 17, pp. 256-262, 2004.

[10] F. Sirois and F. Roy, "Computation of 2D current distribution in superconductors of arbitrary shapes using a new semi-analytical method," IEEE Trans. Appl. Supercond., vol. 17, no. 3, pp. 3836-3845, Sep. 2007.

[11] H. London, "AC losses in superconductors of the second kind," Phys. Lett., vol. 6, pp. 162-165, 1963

[12] R. Hancox, "Calculation of AC losses in a type II superconductor," Proc. Inst. Elect. Eng., vol. 113, no. 7, pp. 1221-1228, Jul. 1966.

[13] W. T. Norris, "Calculation of hysteresis losses in hard superconductors carrying AC: Isolated conductors and edges of thin sheets," J. Phys. D, vol. 3, pp. 489-506, 1970.

[14] F. Gomory and L. Gherardi, "Transport AC losses in round superconducting wire consisting of two concentric shells with different critical current density," Physica C, vol. 280, pp. 151-157, 1997.

[15] B. Douine, J. Leveque, D. Netter, and A. Rezzoug, "Calculation of losses in a SHTc current lead with the help of the dimensional analysis," Physica C, vol. 399, pp. 138-142, 2003.

[16] W. J. Carr, "AC transport current loss in a coated superconductor in the Bean model," Physica C, vol. 415, pp. 109-117, 2004.

[17] J. A. Eikelboom and L. J. M. Van de Klundert, "Hysteresis losses in hollow superconducting filaments and multifilament systems," Cryogenics, vol. 31, pp. 354-362, May 1991.

[18] K. Berger, J. Leveque, D. Netter, B. Douine, and A. Rezzoug, "Influence of temperature and/or field dependence of the E-J power law on trapped magnetic field in bulk YBaCuO," IEEE Trans. Appl. Supercond., vol. 17, no. 2, pp. 3028-3031, Jun. 2007.

[19] J. G. Noudem, L. Porcar, O. Belmont, D. Bourgault, J. M. Barbut, J. Beille, P. Tixador, M. Barrault, and R. Tournier, "Study of the superconducting transition at high pulsed current of bulk Bi-2223 sintered and textured by hot forging," Physica C, vol. 281, pp. 339-344, 1997.

[20] F. Gomory and L. Cesnak, "Loss and magnetization measurement of superconducting magnets pulsed at very low ramp rates," Cryogenics, vol. 25 , no. 7 , pp. $375-380$, July 1985 .

[21] J. Leveque, B. Douine, and D. Netter, "AC losses under self-field in a superconducting tube," in High Temperature Superconductivity 1, Materials, A. V. Narlikar, Ed. New York: Springer-Verlag, 2004, pp. 431-496.

[22] Mathematica 2005 [Online]. Available: http://mathworld.wolfram.com/LambertW-Function.html

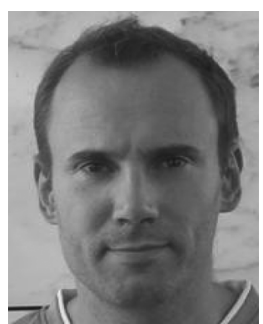

Bruno Douine was born in Montereau, France, in 1966. He received his $\mathrm{Ph}$. D. degree in electrical engineering from University of Nancy, France, in 2001.

$\mathrm{He}$ is currently a Lecturer at the University of Nancy. As member of the Groupe de Recherche en Electrotechnique et Electronique de Nancy, his main subjects of research concern characterization and modelization of superconducting material.

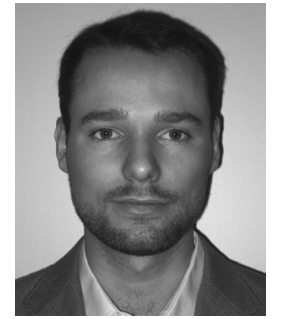

Kévin Berger was born in Montbéliard, France, on January 16, 1980. He received the Ph.D. degree in electrical engineering from Université Henri Poincaré, Nancy, France, in 2006.

$\mathrm{He}$ then joined the Grenoble Electrical Engineering Laboratory (G2Elab) and the Néel Institut, Grenoble in 2006 as a postdoctoral researcher in order to work on superconductor modeling using FLUX software and a conduction cooled SMES. His research interests deal with the design and modeling of superconducting devices like cryomagnets while taking into account coupled magneto-thermal behavior. His research topics also include electrical characterization of superconducting material and $\mathrm{AC}$ loss measurements.

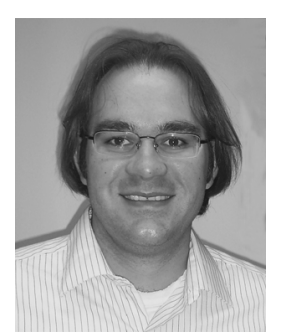

Jules E. Pienkos is working toward the Ph.D. degree in mechanical engineering at Florida State University, Tallahassee, FL. His doctorate research is on the thermal design and cooling of a superconducting motor. His expected graduation date is August 2008.

His research area is in the thermal-fluid sciences and in particular heat transfer. His past work has been on heat exchanger design and experimentation and also superconductivity stability experiments.

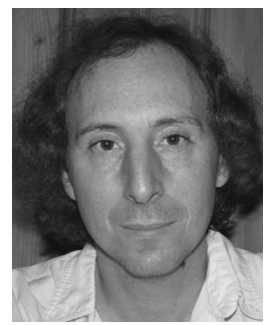

Jean Lévêque was born in Angers, France, in 1963. $\mathrm{He}$ received the $\mathrm{Ph} . \mathrm{D}$. degree in electrical engineering from the University of Grenoble, France, in 1993.

$\mathrm{He}$ is currently Professor at the University of Nancy, France. As member of the Groupe de Recherche en Electrotechnique et Electronique de Nancy, his main subjects of research concern characterization and modelization of superconducting material and applications.

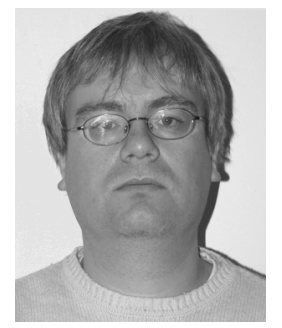

Denis Netter was born in Auchel, France, in 1969 He received the $\mathrm{Ph} . \mathrm{D}$. degree in electrical engineering from the University of Nancy, France, in 1997.

$\mathrm{He}$ is currently a Lecturer at the University of Nancy. As a member of the Groupe de Recherche en Electrotechnique et Electronique de Nancy, his main subjects of research concern superconducting applications and stochastic methods to calculate nonlinear diffusion of the magnetic field in superconducting material. 